\title{
The impact of the Wenchuan earthquake on early puberty: A natural experiment
}

\author{
Qiguo Lian ${ }^{1,2}$, Xiayun Zuo ${ }^{1}$, Yanyan Mao ${ }^{1,2}$, Yan Zhang ${ }^{1,2}$, Shan Luo ${ }^{3}$, Shucheng Zhang ${ }^{4}$, Chaohua Lou ${ }^{1,5}$

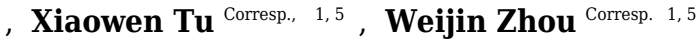 \\ ${ }^{1}$ Key Lab. of Reproduction Regulation of NPFPC, SIPPR, IRD, Fudan University, Shanghai, China \\ 2 School of Public Health, Fudan University, Shanghai, China \\ 3 West China Second University Hospital, Sichuan University, Chengdu, Sichuan, China \\ 4 National Research Institute for Family Planning, Beijing, China \\ 5 Shanghai Institute of Planned Parenthood Research, Shanghai, China \\ Corresponding Authors: Xiaowen Tu, Weijin Zhou \\ Email address: tuxiaowen@sippr.org.cn, zw0822@sina.com
}

Background: The factors influencing pubertal timing have gained much attention due to a secular trend toward earlier pubertal onset in many countries. However, no studies have investigated the association between the great earthquake and early puberty. We aimed to assess whether Wenchuan earthquake is associated with early puberty, in both boys and girls.

Methods: We used data from two circles of a survey on reproductive health in China to explore the impact of Wenchuan earthquake on early puberty , and a total of 9,785 adolescents (4,830 boys, 49.36\%) aged 12-20 years from 29 schools in 8 provinces were recruited. Wenchuan earthquake exposure, defined as those Sichuan students who had not experienced oigarche/menarche before May 12, 2008. Early puberty was identified as a reported onset of oigarche/menarche at 11 years or earlier. We tested the association between Wenchuan earthquake and early puberty in boys and girls. Then subgroup analysis stratified by the age at earthquake exposure also was performed.

Results: In total, 8,883 adolescents (4,543 boys, 51.14\%) with a mean (SD) age of 15.13 (1.81) were included in the final sample. In general, children exposed to earthquake had three times greater risk of early puberty (boys, RR [95\%Cl] =3.18[2.21-4.57]; girls: RR [95\%Cl] =3.16[2.65-3.78]). Subgroup analysis showed that the adjusted RR was 1.90[1.19-3.03] for boys and 2.22[1.75-2.80] for girls. Earthquake exposure predicted almost a fourfold $(\mathrm{RR}[95 \% \mathrm{Cl}]=3.91[1.31-11.72])$ increased risk of early puberty in preschool girls, whereas the increase was about twofold $(\mathrm{RR}[95 \% \mathrm{Cl}]=2.09[1.65-2.64])$ in school girls. Among boys, only older age at earthquake exposure linked to early puberty $(\mathrm{RR}[95 \% \mathrm{Cl}]$ $=1.93[1.18-3.16])$.

Conclusions: Wenchuan earthquake exposure increased the risk of early puberty in boys and girls, and preschoolers were more at risk than schoolchildren. The implications are relevant to support policies for those survivors, especially children, to build back better after disasters. 
1 The impact of the Wenchuan earthquake on early puberty: a natural experiment

2 Qiguo Lian ${ }^{1,2}$ MD, Xiayun Zuo ${ }^{1}$ Ph.D., Yanyan Mao ${ }^{1,2}$ MD, Yan Zhang ${ }^{1,2}$ MD, Shan Luo ${ }^{3}$ Ph.D., 3 Shucheng Zhang ${ }^{4}$ MD, Chaohua Lou ${ }^{1,5}$ MD, Xiaowen Tu ${ }^{1,5}$ Ph.D., Weijin Zhou ${ }^{1,5}$ PhD 4

51 Key Lab. of Reproduction Regulation of NPFPC, SIPPR, IRD, Fudan University, Shanghai, 6 China

72 School of Public Health, Fudan University, Shanghai, China

83 West China Second University Hospital, Sichuan University, Chengdu, Sichuan, China

94 National Research Institute for Family Planning, Beijing, China

105 Shanghai Institute of Planned Parenthood Research, Shanghai, China

12 Corresponding author:

13 Xiaowen Tu (Lead contact)

14 Email: tuxiaowen@sippr.org.cn

15 Phone: 8621-6477-1560

16 Key Lab. of Reproduction Regulation of NPFPC, SIPPR, IRD, Fudan University

17779 Laohumin Road, Shanghai, China, 200237

\section{Weijin Zhou}

Email: zw0822@sina.com

21 Phone: 8621-6477-1603

22 Key Lab. of Reproduction Regulation of NPFPC, SIPPR, IRD, Fudan University

779 Laohumin Road, Shanghai, China, 200237 
ABSTRACT

Background: The factors influencing pubertal timing have gained much attention due to a secular trend toward earlier pubertal onset in many countries. However, no studies have investigated the association between the great earthquake and early puberty. We aimed to assess whether Wenchuan earthquake is associated with early puberty, in both boys and girls.

Methods: We used data from two circles of a survey on reproductive health in China to explore the impact of Wenchuan earthquake on early puberty, and a total of 9,785 adolescents $(4,830$ boys, $49.36 \%$ ) aged $12-20$ years from 29 schools in 8 provinces were recruited. Wenchuan earthquake exposure, defined as those Sichuan students who had not experienced oigarche/menarche before May 12, 2008. Early puberty was identified as a reported onset of oigarche/menarche at 11 years or earlier. We tested the association between Wenchuan earthquake and early puberty in boys and girls. Then subgroup analysis stratified by the age at earthquake exposure also was performed.

Results: In total, 8,883 adolescents (4,543 boys, $51.14 \%)$ with a mean (SD) age of 15.13 (1.81) were included in the final sample. In general, children exposed to earthquake had three times greater risk of early puberty (boys, $\mathrm{RR}[95 \% \mathrm{CI}]=3.18[2.21-4.57]$; girls: $\mathrm{RR}[95 \% \mathrm{CI}]=3.16[2.65-$ 3.78]). Subgroup analysis showed that the adjusted RR was 1.90[1.19-3.03] for boys and 2.22[1.75-2.80] for girls. Earthquake exposure predicted almost a fourfold (RR [95\%CI]

$41=3.91[1.31-11.72])$ increased risk of early puberty in preschool girls, whereas the increase was about twofold $(\mathrm{RR}[95 \% \mathrm{CI}]=2.09[1.65-2.64])$ in schoolgirls. Among boys, only older age at earthquake exposure linked to early puberty $(\mathrm{RR}[95 \% \mathrm{CI}]=1.93[1.18-3.16])$.

Conclusions: Wenchuan earthquake exposure increased the risk of early puberty in boys and girls, 
45 and preschoolers were more at risk than schoolchildren. The implications are relevant to support

46 policies for those survivors, especially children, to build back better after disasters.

47 Key words: Wenchuan earthquake; Early Puberty; Natural disasters 


\section{Background}

50

The pubertal timing is of great interest to both health providers and the public. There's evidence that early puberty is associated with a broad range of short- and long-term adverse health consequences, especially for adolescent girls(Stroud \& Davila 2016). The physical and emotional risks of early maturation include not only depression, anxiety, substance abuse and early sexual debut during adolescence(Mensah et al. 2013; Walvoord 2010; William Copeland et al. 2010), but also reproductive cancers and metabolic syndrome in later life(Day et al. 2015; Walvoord 2010; William Copeland et al. 2010). Pubertal timing is a complex trait that affected by the geneenvironment interactions(Karapanou \& Papadimitriou 2010). A deeper understanding of the potential contributors to pubertal timing is essential due to a secular trend toward earlier pubertal onset in many countries (Papadimitriou 2016; Sørensen et al. 2012).

In addition to chemical exposures and obesity, emotional stress is another culprit that could jumpstart puberty earlier (Ellis \& Garber 2000; Rickard et al. 2014; Sun et al. 2017). According to psychosocial acceleration theory, an evolutionary-biological perspective on human development, differences in environmental contexts tend to channel children into divergent developmental trajectories on pubertal timing (Belsky et al. 1991; Webster et al. 2014). The stresses caused by harsh environments regulate reproductive strategies more susceptible to enhance reproductive fitness and maximize the probability of leaving descents(Pesonen et al. 2008), including early pubertal timing (Belsky 2012; Webster et al. 2014). There is considerable evidence that childhood abuse, family conflict, father absence, and parental mental disorder are associated with earlier puberty in girls (Ellis \& Garber 2000; Rickard et al. 2014). A cohort study also linked household 
70 socioeconomic disadvantage to earlier pubertal timing in both boys and girls (Sun et al. 2017).

71 War is another harsh environmental stressor that could influence the pubertal timing (Belsky

72 2008). The 1934-1944 Helsinki Birth Cohort Study in Finland revealed that former Helsinki

73 evacuated girls who were sent to live foster families in Sweden and Denmark during world war

74 II had earlier menarche than did the non-separated cohort members who remained at home(Pesonen et al. 2008). Besides, hurricane and earthquake are two of the most common deadly types of natural disasters that could risk the physical and mental health of the child survivors both short-term and long-term (Marsee 2008; Zheng et al. 2012). Based on psychosocial acceleration theory, the disasters related stressors could affect the pubertal timing(Belsky et al. 1991; Webster et al. 2014). However, no study has investigated the association between the earthquake and early puberty. The 8.0-magnitude Wenchuan earthquake that occurred on May 12, 2008, in Sichuan Province was the biggest and deadliest earthquake since the 1950 Tibet earthquake and 1976 Tangshan earthquake in China. This disaster impacted over 15 million people, rendered at least 4.8 million homeless, and left 69,197 people dead, 374,176 injured and 18,222 listed as missing(Fan et al. 2017). The disaster generated a natural experimental setting for measuring the impact of the earthquake for early puberty among the child survivors of the quake. We hypothesized that exposure to Wenchuan earthquake was associated increased risk of early puberty, with younger age at exposure higher risk of early puberty.

\section{Methods}

\section{Study design and participants}


91 The data in the current study are drawn from 2013 and 2015 cycles of a multisite survey on the

92 reproductive health among Chinese middle school students aged 12-20 years from 29 schools in 8

93 provinces. The 2013 survey included Sichuan, Hebei, Shaanxi, Shandong, Heilongjiang, and

94 Guangxi, while 2015 survey sampled Sichuan, Anhui and Inner Mongolia. The data was collected

95 electronically in student computer labs (Lian et al. 2018; Mao et al. 2018), including age at

96 menarche/ejaculation, knowledge, attitude and health risk behaviors about reproductive health. We

97 investigated 9,785 adolescents (4,830 boys and 4,955 girls) in two cycles of the survey and dropped

98902 adolescents who had experienced oigarche/menarche before Wenchuan earthquake because

99 their pubertal timing accessed by oigarche/menarche could not be influenced by the earthquake.

100 The final sample for analysis included 8,883 adolescents (4,534 boys and 4,340 girls).

101 The institutional review board of Shanghai Institute of Planned Parenthood Research reviewed and

102 approved this study (2012-01). Written consents were obtained from both parent and child before

103 the survey.

104 Measures

105 Earthquake exposure

106 For the present study, which was not originally designed to estimate the individual-level exposure

107 to Wenchuan earthquake, we labeled the students lived in Sichuan as a nature indicator of the

108 Wenchuan earthquake exposure, and their peers in other provinces as a non-exposure group. The

109 Sichuan students were sampled from Wenchuan country, Mianzhu city, Deyang City, Luojiang

110 country, and Zhongjiang country. The distance from the quake's epicenter (kilometers) is 9.60,

$11194.23,100.94,130.76$ and 147.76 respectively. 


\section{Early puberty}

113 We measured pubertal timing by age at first ejaculation(oigarche) for boys or period(menarche)

114 for girls and defined early puberty as a reported onset of oigarche/menarche at 11 years or

115 earlier(Kaltiala-Heino et al. 2011). Oigarche and menarche can be used to measure the pubertal

116 maturation for boys and girls respectively in survey studies, although the self-reported data have

117 been criticized(Dick et al. 2000). Many studies preferred to use Tanner stages, evaluated by

118 either trained clinicians or children themselves(Hayatbakhsh et al. 2009), however, there is

119 evidence that age at oigarche/menarche and Tanner stages produced consistent results(Kaltiala-

120 Heino et al. 2011).

\section{Confounders}

122 We identified potential confounders by drawing causal acyclic graphs (DAGs) based on prior

123 knowledge in the exposure-outcome association(Foraita et al. 2014). In this natural experiment, it

124 appears that there are no essential confounders. Many factors, including body mass index (BMI),

125 socioeconomic status, family structure, and maternal age of menarche were not considered to be

126 potential confounders because they had no a priori association with Wenchuan earthquake

127 exposure.

128 Statistical analysis

129 Univariate statistics were used to describe the mean age, the frequencies of the individual level

130 and macro level characteristics, and the rate of early puberty. We examined the association of

131 Wenchuan earthquake exposure with early oigarche and early menarche and calculated the

132 strength of associations that measured by rate ratio (RR) with $95 \%$ confidential interval 
133 (CI)(Cummings 2009). We also computed RRs and 95\% CIs for boys and girls respectively. We

134 also performed subgroup analysis on the age group when children exposed to Wenchuan

135 earthquake. The age at earthquake was recoded as younger(preschoolers, age $<7$ years) and older 136 group(schoolchildren, age $\geq 7$ years).

137 We estimated the strength of association of earthquake exposure with RR because this natural experiment can be understood as a retrospective cohort study that earthquake exposure was identified from the recorded information. Statistical analysis used Stata/SE, version 15.1

140 (StataCorp LLC).

\section{Role of the funding source}

142 The funders of the study had no role in study design, data collection, data analysis, data interpretation, or writing of the manuscript. The corresponding authors had full access to all the

144 data in the study and had final responsibility for the decision to submit for publication

\section{Results}

A total of 8,883 adolescents from 29 schools in 8 provinces were included in the analysis, of which 4,543 were boys (51.14\%), and 4,340 were girls (48.86\%) (Table 1$)$. The mean age the final pooled sample was 15.15 years (standard deviation $[\mathrm{SD}]=1.81$ ). There were eight schools in Sichuan exposed to Wenchuan earthquake, and 21 schools in other provinces unexposed. In the final sample, there were 2,658 earthquake survivors and 6,225 adolescents who did not experience Wenchuan earthquake. Nearly $14 \%$ of the participants were preschoolers and the rest $86 \%$ were schoolchildren when Wenchuan earthquake struck.

The rate of the early puberty was $2.55 \%$ for boys and 10.14 for girls (Table 2 ). As illustrated in 
154 Table 3, the rate of early oigarche was $4.9 \%$ for male earthquake survivors and $1.54 \%$ for boys

155 who did not experience Wenchuan earthquake. The RR of the association between earthquake 156 exposure and early oigarche was $3.18[95 \% \mathrm{CI}, 2.21-4.57, \mathrm{p}<0.001]$. Similarly, Table 3 also 157 presents the rate of early menarche (19.50\% for exposure and $6.17 \%$ for non-exposure) and the

RR between earthquake exposure and early menarche (3.16[2.65-3.78], $\mathrm{p}<0.001)$.

We conducted subgroup analysis for male and female respectively, given that the children's age at earthquake may serve as an effect-measure modifier on the association between earthquake exposure and early puberty (Table 4). The earthquake exposure appeared to double the risk of early puberty(boys:adjusted $\mathrm{RR}=1.90[1.19-3.03], \mathrm{P}<0.050$; girls: 2.22[1.75-2.80], $\mathrm{P}<0.050$ ). As shown in Table 4, older age at earthquake was a protective factor that reduced the risk of early menarche more than 50\% compared with younger age at Wenchuan earthquake (preschool girls: $R R=3.91[1.31-11.72], p=0.003$; schoolgirls: $R R=2.09[1.65-2.64], p<0.001)$. The association was non-significant among adolescent boys who exposed to Wenchuan earthquake before age seven years $(\mathrm{RR}=1.73[0.43-6.91], \mathrm{p}=0.425)$, but significant among the survivors of schoolboys $(\mathrm{RR}=1.93$ [1.18-3.16], $\mathrm{p}=0.007)$

\section{Discussion}

To our best knowledge, this is the first study to investigate the potential impacts of the great earthquake on pubertal timing. The 2008 Wenchuan earthquake offers a unique opportunity to explore the effects of the earthquake on the quake survivors' later life. In this natural experiment, exposure to Wenchuan earthquake doubled the risk ratio of early puberty (1.90 for boys, 2.22 for girls). What's more, there may be effect modification by children's age at earthquake exposure. 
175 Earthquake exposure predicted almost a fourfold increased risk ratio of early puberty in preschool

176 girls, whereas the increase was about twofold in schoolgirls. Similarly, earthquake exposure

177 doubled the risk ratio in schoolboys. However, the association of earthquake exposure with early

178 puberty was not statistically significant in preschool boys. The main reason for the unanticipated

179 result was the sample was too small (Unexposed $=50[$ Cases $=2])$.

180 Factors that influence pubertal maturation have gained much attention recently because of the

181 secular trend to earlier puberty(Karapanou \& Papadimitriou 2010). There are many determinants

182 of early puberty, but none of these servers as potential confounders in the present study because

183 Wenchuan earthquake was apparently not affected by any of these determinants. The age at

earthquake exposure might mediate the impact of earthquake exposure on early puberty as an

intermediary step in the causal pathway. On the one hand, younger age at earthquake exposure

could prolong the time of the earthquake shock action. Children who experienced earthquake

earlier, on the other hand, may suffer more from earthquake-related stress and trauma, which put

them at higher risk for earlier puberty. We found that preschool students were more vulnerable to

early puberty when they were exposed to earthquake, which made a new contribution to

psychosocial acceleration theory that the first 5-7 years of life might be especially influential in

shaping reproductive strategy.

In the opinion of evolutionary-mind thinkers, accelerated somatic development was adaptive under

dangerous or unpredictable environments because this tactic increased the chances of dispersing

genes across generations before dying (Belsky 2012; Belsky et al. 1991). The adaptive 
196 197

individuals in modern society, including early menarche, early sexual debut, and risktaking(Belsky 2012; Ellis \& Bjorklund 2012).

Considerable research on adverse health consequences of disaster events including earthquakes and hurricanes focused on mental health (Carroll \& Frakt 2017; Fan et al. 2017; Shultz \& Galea 2017). Our findings revealed the profound impacts of the earthquake on early puberty both in boys and girls. A better understanding of this impacts could inform the post-disaster interventions, which is crucial to the well-being of survivors, especially children (Carroll \& Frakt 2017). Given that children take their cues from adults, parents/guardians/caretakers should act calmly and confidently when disasters occur(Carroll \& Frakt 2017). Pediatricians should screen the health conditions of the young survivors of disasters in the years to come. Besides, policies that provide the children with supportive environments and caring relationships are critical in rebuilding their lives after disasters.

The underlying biological mechanisms that earthquake might influence the early puberty are still barely understood. The stressors, including early childhood adversity and earthquake exposure, can affect not only the hypothalamic-pituitary-adrenal (HPA) and hypothalamic-pituitary-gonadal (HPG) axes individually, but the cross-talk between the two axes, possibly mediating HPA-HPG coupling(Ruttle et al. 2015; Shirtcliff et al. 2015). The mechanism explains how stressors may trigger pubertal development. Animal studies also suggested that social environment could influence the HPG axis at the molecular level(Maruska \& Fernald 2011). However, the role of genomic changes regulating pubertal development remains unclear(Sun et al. 2017) and is a promising field (Lomniczi \& Ojeda 2016). Studies also suggested that major adversity in early 
217 childhood could impair the brain's reward circuits processing threat and stress(Boyce et al. 2012;

218 Hanson et al.), which might influence the regulation of reproductive hormones, resulting in early-

219 onset puberty (Boyce et al. 2012).

220 Strengths and limitations

221 The primary strength of this study is the clear temporal relationship between Wenchuan earthquake

exposure and early puberty, which improved our ability to make a valid causal inference. To avoid

temporal ambiguity, we excluded individuals who had experienced oigarche/menarche before May

12,2008 . Also, this study took advantage of the natural experiment generated by the Wenchuan

earthquake to investigate the long-term effect of the great earthquake on early puberty.

Our study has several limitations worth noting. First, the earthquake exposure was not measured

at an individual level that could have allowed the higher accuracy of exposure estimates. There

was not any information on death or illness caused by the earthquake in their family members in

the present study. Second, we failed to investigate the association of severity of exposure with

earlier puberty due to the small sample size in the most damaged areas (237 in Wenchuan and 303

in Mianzhu). Third, the data on oigarche/menarche were collected by self-report and thus may be

prone to recall bias. However, there is much evidence to show a high degree of validity in recalled

age at menarche, especially among adolescents (Carol et al. 2001; Koo \& Rohan 1997; Malina et

al. 2015; Mao et al. 2018). Fourth, we did not use Kaplan-Meier curve or Cox proportional hazards

modeling because of lacking the data on the full spectrum of ages at puberty. It would be much

more informative to show the difference in age at puberty curves graphically. Fifth, the study is

susceptible to confounding by time trend, which could have been controlled if we could build in a 
238 before/after comparison in both exposed and unexposed communities. However, it impossible to 239 collect data pre-earthquake because of the unpredictable nature of earthquake(Fan et al. 2017).

240 Last, we did not collect other factors that influencing the puberty, including BMI, maternal BMI,

241 and early maternal menarche, to ensure the comparability between the exposure and non-exposure

242 groups, which could introduce selection bias and hinder the ability to generalize our findings to a

243 more diverse population.

244 Conclusions

245 Exposure to Wenchuan earthquake elevated the risk of early puberty in both boys and girls, and 246 preschoolers are more vulnerable to earthquake compared with schoolchildren. The findings

247 suggest that the health of children, especially younger children, must remain a focus in the recovery 248 from disasters.

\section{Acknowledgments}

250 The authors thank site coordinators and the students involved for their support. The authors also 251 acknowledge the advice from Dr. Qianxi Zhu. 
253

254

255

256

257

258

259

260

261

262

263

264

265

266

267

268

269

270

271

272

273

274

275

276

277

278

279

280

281

282

283

284

285

286

287

288

289

290

291

292

\section{References}

Belsky J. 2008. War, trauma and children's development: Observations from a modern evolutionary perspective. International Journal of Behavioral Development 32:260-271. 10.1177/0165025408090969

Belsky J. 2012. The Development of Human Reproductive Strategies. Current Directions in Psychological Science 21:310-316. 10.1177/0963721412453588

Belsky J, Steinberg L, and Draper P. 1991. Childhood Experience, Interpersonal Development, and Reproductive Strategy: An Evolutionary Theory of Socialization. Child Dev 62:647-670. 10.2307/1131166

Boyce WT, Sokolowski MB, and Robinson GE. 2012. Toward a new biology of social adversity. Proceedings of the National Academy of Sciences 109:17143-17148. 10.1073/pnas.1121264109

Carol K, J. CR, and Leslie B. 2001. Ability of Young Women to Recall Past Body Size and Age at Menarche. Obesity Research 9:478-485. doi:10.1038/oby.2001.62

Carroll AE, and Frakt AB. 2017. Children's health must remain a focus in the recovery from hurricane harvey. JAMA Pediatrics. 10.1001/jamapediatrics.2017.3851

Cummings P. 2009. Methods for estimating adjusted risk ratios. Stata Journal 9:175-196.

Day FR, Elks CE, Murray A, Ong KK, and Perry JR. 2015. Puberty timing associated with diabetes, cardiovascular disease and also diverse health outcomes in men and women: the UK Biobank study. Sci Rep 5:11208. 10.1038/srep11208

Dick DM, Rose RJ, Viken RJ, and Kaprio J. 2000. Pubertal timing and substance use: associations between and within families across late adolescence. Dev Psychol 36:180-189.

Ellis BJ, and Bjorklund DF. 2012. Beyond mental health: an evolutionary analysis of development under risky and supportive environmental conditions: an introduction to the special section. Developmental Psychology 48:591-597. 10.1037/a0027651

Ellis BJ, and Garber J. 2000. Psychosocial antecedents of variation in girls' pubertal timing: maternal depression, stepfather presence, and marital and family stress. Child Dev 71:485-501.

Fan F, Zhou Y, Mo L, Zhang W, Xie J, and Liu X. 2017. Cohort Profile: The Wenchuan Earthquake Adolescent Health Cohort Study. Int J Epidemiol 46:27-28. 10.1093/ije/dyw013

Foraita R, Spallek J, and Zeeb H. 2014. Directed Acyclic Graphs. In: Ahrens W, and Pigeot I, eds. Handbook of Epidemiology. New York, NY: Springer New York, 1481-1517.

Hanson JL, Hariri AR, and Williamson DE. Blunted Ventral Striatum Development in Adolescence Reflects Emotional Neglect and Predicts Depressive Symptoms. Biological Psychiatry 78:598-605. 10.1016/j.biopsych.2015.05.010

Hayatbakhsh MR, Najman JM, McGee TR, Bor W, and O'Callaghan MJ. 2009. Early pubertal maturation in the prediction of early adult substance use: a prospective study. Addiction 104:59-66. 10.1111/j.13600443.2008.02382.x

Kaltiala-Heino R, Koivisto A-M, Marttunen M, and Fröjd S. 2011. Pubertal Timing and Substance Use in Middle Adolescence: A 2-Year Follow-up Study. J Youth Adolesc 40:1288. 10.1007/s10964-011-9667-1

Karapanou O, and Papadimitriou A. 2010. Determinants of menarche. Reproductive Biology and Endocrinology 8:115. 10.1186/1477-7827-8-115

Koo MM, and Rohan TE. 1997. Accuracy of short-term recall of age at menarche. Ann Hum Biol 24:61-64.

Lian Q, Su Q, Li R, Elgar FJ, Liu Z, and Zheng D. 2018. The association between chronic bullying victimization with 
weight status and body self-image: a cross-national study in 39 countries. PeerJ 6:e4330. 10.7717/peerj.4330

Lomniczi A, and Ojeda SR. 2016. The Emerging Role of Epigenetics in the Regulation of Female Puberty. Endocr Dev 29:1-16. 10.1159/000438840

Malina RM, Rogol AD, Cumming SP, Coelho e Silva MJ, and Figueiredo AJ. 2015. Biological maturation of youth athletes: assessment and implications. British Journal of Sports Medicine 49:852-859. 10.1136/bjsports2015-094623

Mao Y, Lian Q, Zuo X, Zhang Y, Luo S, Zhang S, Tu X, Lou C, and Zhou W. 2018. Validity of self-reported age at menarche in computer-assisted interview among Chinese schoolgirls: a cross-sectional study. BMJ Open 8:e016799. 10.1136/bmjopen-2017-016799

Marsee MA. 2008. Reactive Aggression and Posttraumatic Stress in Adolescents Affected by Hurricane Katrina. Journal of Clinical Child \& Adolescent Psychology 37:519-529. 10.1080/15374410802148152

Maruska KP, and Fernald RD. 2011. Social Regulation of Gene Expression in the Hypothalamic-Pituitary-Gonadal Axis. Physiology 26:412-423. 10.1152/physiol.00032.2011

Mensah FK, Bayer JK, Wake M, Carlin JB, Allen NB, and Patton GC. 2013. Early Puberty and Childhood Social and Behavioral Adjustment. Journal of Adolescent Health 53:118-124. 10.1016/j.jadohealth.2012.12.018

Papadimitriou A. 2016. Timing of Puberty and Secular Trend in Human Maturation. In: Kumanov P, and Agarwal A, eds. Puberty: Physiology and Abnormalities. Cham: Springer International Publishing, 121-136.

Pesonen A-K, Räikkönen K, Heinonen K, Kajantie E, Forsén T, and Eriksson JG. 2008. Reproductive traits following a parent-child separation trauma during childhood: A natural experiment during World War II. American Journal of Human Biology 20:345-351. 10.1002/ajhb.20735

Rickard IJ, Frankenhuis WE, and Nettle D. 2014. Why Are Childhood Family Factors Associated With Timing of Maturation? A Role for Internal Prediction. Perspectives on Psychological Science 9:3-15. $10.1177 / 1745691613513467$

Ruttle PL, Shirtcliff EA, Armstrong JM, Klein MH, and Essex MJ. 2015. Neuroendocrine coupling across adolescence and the longitudinal influence of early life stress. Developmental psychobiology 57:688-704. 10.1002/dev.21138

Shirtcliff EA, Dismukes AR, Marceau KP, Ruttle P, Simmons J, and Han G. 2015. A dual-axis approach to understanding neuroendocrine development. Developmental psychobiology 57:643-653. 10.1002/dev.21337

Shultz JM, and Galea S. 2017. Mitigating the mental and physical health consequences of hurricane harvey. JAMA. 10.1001/jama.2017.14618

Sørensen K, Mouritsen A, Aksglaede L, Hagen CP, Mogensen SS, and Juul A. 2012. Recent Secular Trends in Pubertal Timing: Implications for Evaluation and Diagnosis of Precocious Puberty. Hormone Research in Paediatrics 77:137-145.

Stroud CB, and Davila J. 2016. Pubertal Timing. In: Levesque RJR, ed. Encyclopedia of Adolescence. Cham: Springer International Publishing, 1-9.

Sun Y, Mensah FK, Azzopardi P, Patton GC, and Wake M. 2017. Childhood Social Disadvantage and Pubertal Timing: A National Birth Cohort From Australia. Pediatrics 139:e20164099. 10.1542/peds.2016-4099

Walvoord EC. 2010. The Timing of Puberty: Is It Changing? Does It Matter? Journal of Adolescent Health 47:433439. 10.1016/j.jadohealth.2010.05.018

Webster GD, Graber JA, Gesselman AN, Crosier BS, and Schember TO. 2014. A Life History Theory of Father Absence and Menarche: A Meta-Analysis. Evolutionary Psychology 12:147470491401200202. 
$10.1177 / 147470491401200202$

William Copeland, Lilly Shanahan, Shari Miller, E. Jane Costello, Adrian Angold, and Barbara Maughan. 2010. Outcomes of Early Pubertal Timing in Young Women: A Prospective Population-Based Study. American Journal of Psychiatry 167:1218-1225. 10.1176/appi.ajp.2010.09081190

Zheng S, Fu W, Zhou J, Dong X, Liu Z, Wang Y, and Zhang Q. 2012. Prevalence and related factors of irritable bowel syndrome among middle-school students in areas affected by Wenchuan Earthquake: an epidemiological study. J Clin Gastroenterol 46:345-346. 10.1097/MCG.0b013e31824712d0 


\section{Table 1 (on next page)}

Sample characteristics by survey cycle, n (\%) 
1 Table 1 Sample characteristics by survey cycle, $\mathrm{n}(\%)$

\begin{tabular}{|c|c|c|c|}
\hline \multirow[b]{2}{*}{ Characteristics } & \multicolumn{2}{|c|}{ Survey cycle } & \multirow{2}{*}{$\begin{array}{l}\text { Total }(n=8,883 \\
)\end{array}$} \\
\hline & $\begin{array}{l}\text { 2013, } 5 \text { years after the } \\
\text { earthquake }(n=5,417)\end{array}$ & $\begin{array}{l}2015,7 \text { years after the } \\
\text { earthquake }(n=3,466)\end{array}$ & \\
\hline Age, Mean(SD) & $15.15(1.78)$ & $15.11(1.86)$ & $15.13(1.81)$ \\
\hline \multicolumn{4}{|l|}{ Sex } \\
\hline Male & $2,849(52.59)$ & $1,694(48.87)$ & $4,543(51.14)$ \\
\hline Female & $2,568(47.41)$ & $1,772(51.13)$ & $4,340(48.86)$ \\
\hline \multicolumn{4}{|c|}{ Wenchuan earthquake } \\
\hline Exposure & $967(17.85)$ & $1,691(48.79)$ & $2,658(29.92)$ \\
\hline Non-exposure & $4,450(82.15)$ & $1,775(51.21)$ & $6,225(70.08)$ \\
\hline \multicolumn{4}{|l|}{ Cities } \\
\hline Exposure & 1 & 1 & 1 \\
\hline Non-exposure & 5 & 2 & 7 \\
\hline \multicolumn{4}{|l|}{ Schools } \\
\hline Exposure & 2 & 6 & 8 \\
\hline Non-exposure & 15 & 6 & 21 \\
\hline
\end{tabular}

2 


\section{Table 2 (on next page)}

Sample characteristics by sex, $\mathrm{n}(\%)$ 
1 Table 2 Sample characteristics by sex, $\mathrm{n}(\%)$

\begin{tabular}{llll}
\hline Characteristics & Boys $(n=4,543)$ & Girls $(n=4,340)$ & Total $(n=8,883)$ \\
\hline $\begin{array}{l}\text { Age, Mean(SD) } \\
\text { Wenchuan earthquake }\end{array}$ & $15.16(1.84)$ & $15.10(1.77)$ & $15.13(1.81)$ \\
$\quad$ Exposure & $1,366(30.07)$ & $1,292(29.77)$ & $2,658(29.92)$ \\
$\quad$ Non-exposure & $3,177(69.93)$ & $3,048(70.23)$ & $6,225(70.08)$ \\
Early puberty & & & \\
$\quad$ Yes & $116(2.55)$ & $440(10.14)$ & $556(6.26)$ \\
No & $4,427(97.45)$ & $3,900(89.86)$ & $8,327(93.74)$ \\
Age at earthquake & & & $1,203(13.54)$ \\
$\quad<7$ years & $617(13.58)$ & $586(13.50)$ & $7,680(86.46)$ \\
$\quad \geq 7$ years & $3,926(86.42)$ & $3,754(86.50)$ & \\
\hline
\end{tabular}

2 


\section{Table 3(on next page)}

The crude association between Wenchuan earthquake and early puberty 
1 Table 3 The crude association between Wenchuan earthquake and early puberty

\begin{tabular}{cllll}
\hline \multirow{2}{*}{ Early puberty } & \multicolumn{2}{l}{ Wenchuan earthquake, $\mathrm{n}(\%)$} & \multirow{2}{*}{ Crude RR(95\% Cl) } & P value \\
\cline { 2 - 5 } & Exposure & Non-exposure & $3.18(2.21-4.57)$ & $\mathrm{p}<0.001$ \\
\hline Boys & & & & \\
Yes & $67(4.90)$ & $49(1.54)$ & & \\
No & $1,299(95.10)$ & $3,128(98.46)$ & $3.16(2.65-3.78)$ & $\mathrm{p}<0.001$ \\
Girls & & & & \\
Yes & $252(19.50)$ & $188(6.17)$ & & \\
No & $1,040(80.50)$ & $2,860(93.83)$ & & \\
\hline
\end{tabular}

2 


\section{Table 4 (on next page)}

The association between Wenchuan earthquake and early puberty, by age at earthquake 
1 Table 4 The association between Wenchuan earthquake and early puberty, by age at earthquake

\begin{tabular}{lllll}
\hline & \multicolumn{2}{c}{ Boys } & \multicolumn{2}{c}{ Girls } \\
\hline & $\mathrm{n}$ & $\mathrm{RR}(95 \% \mathrm{Cl}, \mathrm{p}$ value $)$ & $\mathrm{n}$ & $\mathrm{RR}(95 \% \mathrm{Cl}, \mathrm{p}$ value $)$ \\
\hline $\begin{array}{l}\text { Age at earthquake } \\
\quad \text { 7 years }\end{array}$ & 617 & $1.73(0.43-6.91, \mathrm{p}=0.425)$ & 586 & $3.91(1.31-11.72, \mathrm{p}=0.003)$ \\
$\quad$ 7 years & 3,926 & $1.93(1.18-3.16, \mathrm{p}=0.007)$ & 3,754 & $2.09(1.65-2.64, \mathrm{p}<0.001)$ \\
Crude & 4,543 & $3.18(2.21-4.57, \mathrm{p}<0.001)$ & 4,340 & $3.16(2.65-3.78, \mathrm{p}<0.001)$ \\
M-H combined $^{*}$ & 4,543 & $1.90(1.19-3.03, \mathrm{P}<0.050)$ & 4,340 & $2.22(1.75-2.80, \mathrm{P}<0.050)$ \\
\hline
\end{tabular}

